\title{
Cambiando paradigmas en cirugía: Creación del Departamento de Cirujanas y Promoción de Buenas Prácticas en Entrevistas
}

\author{
Changing Paradigms in Surgery; Creation of the Female Surgeons Department \\ and Promotion of Good Practices in Interviews
}

\author{
Claudia Albornoz Garrido ${ }^{1}$
}

Los tiempos han cambiado y la presencia de mujeres en todas las áreas laborales es una realidad. Las escuelas de medicina en Chile desde el año 2018 tienen más egresadas mujeres que hombres. El escenario actual de las cirujanas como una minoría en Chile (solo 15\% del total) también cambiará en el mediano plazo, dado que en la actualidad más del $30 \%$ de las residentes son mujeres. Existen cirujanas en los servicios de urgencia, en atención primaria, en lugares extremos, y en todas las subespecialidades $^{1}$. Somos parte de los equipos de trabajo, entonces, ¿por qué resistirse al cambio?

Un equipo de salud más diverso permite entregar una mejor calidad de atención. Las mujeres tenemos una serie de características positivas: estamos acostumbradas a cuidar de los demás, a formar equipos $\mathrm{y}$ a ser el agente aglutinante en nuestra familia, entorno y también en el trabajo. Nuestra capacidad de trabajo es infinita, de hecho, después de terminar nuestro trabajo remunerado seguimos trabajando en nuestros hogares. Estudios demuestran que las mujeres somos más críticas y exigentes con nosotras mismas: Lind et al., demostraron que cuando se les pedía a estudiantes de sexo femenino que se autoevaluaran en una rotación de cirugía, ellas se asignaban menores calificaciones que sus pares masculinos, aunque tenían un mejor desempeño de acuerdo con la evaluación de sus profesores ${ }^{2}$. Debido a que durante nuestra formación hemos estado generalmente en desventaja, hemos tenido que estudiar más, prepararnos más, porque se nos exige más.

La creación del Departamento de Cirujanas en el año 2020 obedeció al deseo de un grupo de cirujanas de poder tener más representación, visibilidad y posibilidades de desarrollo dentro de la Sociedad de Cirujanos (que, a mi juicio, debe cambiar el nombre a Sociedad de Cirugía acorde al avance de los tiem- pos). Sabemos que la necesidad de tener un departamento especial para las Cirujanas puede producir desconcierto en algunos cirujanos, pero el objetivo no es desunir sino lograr insertar a las cirujanas de lleno en nuestra Sociedad y potenciar su desarrollo científico. Pese a estar constituido hace menos de un año, el Departamento de Cirujanas ya ha desarrollado varias actividades científicas, como la Seccional en el Congreso 2020 con invitadas(os) de primer nivel y con alta convocatoria, curso de Introducción a la Investigación, actividades con Academias Científicas para acercar la Cirugía a las alumnas que pueden estar considerando una especialidad quirúrgica, el primer debate presidencial de cara a las elecciones del último directorio y múltiples otras actividades en las que seguiremos promoviendo activamente el crecimiento científico de la Sociedad.

Sin embargo, el departamento también tiene un rol en la educación acerca de buenas prácticas entre miembros de la Sociedad y hacia nuestras residentes, alumnas y futuras residentes. Existen aún conductas discriminatorias que deben ser erradicadas, como es el caso de entrevistas sesgadas por género a postulantes a un programa de especialización en cirugía. Varias universidades han implementado protocolos para las entrevistas de manera que no se pregunten algunos temas que pueden ser considerados discriminatorios. En el caso de las mujeres, lamentablemente aún muchas veces se les pregunta más acerca de planes de maternidad, estado civil, quién va a cuidar a los niños mientras ellas estén en formación, que acerca de sus intereses, méritos y currículum ${ }^{3}$. Estas preguntas rara vez son realizadas a los postulantes hombres, por lo que constituye una discriminación de género. Debemos erradicar el concepto de que la cirugía es incompatible con la maternidad o la formación de una familia. Para na-
'Presidenta del Departamento de Cirujanas.

Correspondencia a: Dra. Claudia Albornoz Garrido clau.albornoz@gmail.com 
die es un misterio que las cirujanas estamos en edad fértil en nuestro período de formación; sin embargo, las mujeres somos las que tenemos a los niños que serán el soporte de la sociedad y la economía más adelante.

El Ministerio de Salud firmó el $1^{\circ}$ de diciembre de 2017 un acuerdo con el Colegio Médico, las Facultades de Medicina de la Universidad de Chile, Pontificia Universidad Católica de Chile, Universidad de Santiago, Universidad de Valparaíso, Universidad Diego Portales, Universidad Católica del Norte, Universidad de Antofagasta, Universidad Mayor, Universidad de Concepción, Universidad Andrés Bello, Universidad de los Andes, Universidad del Desarrollo, Universidad San Sebastián, Universidad Austral de Chile, Universidad Católica del Maule, Universidad Finis Terrae, Universidad Católica de la Santísima Concepción, Universidad de la Frontera, Universidad de Talca, Universidad Autónoma, la Agrupación de Médicos Generales de Zona y la Agrupación de Estudiantes de Medicina de Chile, un Protocolo de Acuerdo que regula la habilitación para el ingreso a las especialidades, en el que se establece que ciertos temas $\mathrm{NO}$ deben ser abordados en las etapas de evaluación: "estado civil del postulante, planificación familiar (maternidad o paternidad), presencia de hijos o configuración de familia, orientación religiosa, postura frente a temas valóricos (ej., aborto, eutanasia), orientación política o militancia, nivel socioeconómico, orientación sexual o identidad de género, etnia y establecimiento de educación secundaria de procedencia" $(\text { sic })^{4}$. El Servicio Civil, órgano del Estado que busca ser un referente en materias de empleo y calidad de vida laboral, establece lineamentos similares en su Código de Buenas Prácticas Laborales 5 . Por lo tanto, cualquier pregunta de este tipo en un proceso de selección para un programa de especialización en Cirugía, además de ser discriminatoria, estará faltando a la norma y es potencialmente sancionable.

Es hora de dejar atrás este tipo de actitudes. No tengamos miedo al cambio, y participemos entre todos en la creación de una sociedad más justa. Por sus hijas, sus hermanas y sus esposas.

\section{Bibliografía}

1. Albornoz Garrido C, Carrillo González $\mathrm{K}$, Vargas Vargas V, Pedrero Bustamante $\mathrm{P}$, Fernández Albornoz M, Fonseca Salamanca V, et al. Visibilizando a las Cirujanas en Chile. Rev. Cirugia. 2020;73(4). Disponible en: doi: 10.35687/ s2452-45492021004976.

2. Lind DS, Rekkas S, Bui V, Lam T,
Beierle E, Copeland EM. Competencybased student self-assessment on a surgery rotation. J Surg Res. 2002;105:31-4.

3. Valenzuela M. La mujer cirujano: todavía un cuento para "niñas rebeldes?". Rev Cir $2020 ; 72: 7-8$

4. Subsecretaría de Redes Asistenciales, Ministerio de Salud de Chile. Protocolo de Acuerdo Habilitación para el ingreso a la especialidad. https://medicina.udd.
cl/files/2020/03/PROTOCOLO-DEACUERDO-HABILITACIONES.pdf (Consultado el 10 de mayo de 2021).

5. Servicio Civil. Código de buenas prácticas laborales sobre no discriminación para la Administración Central del Estado; https://documentos.serviciocivil. $\mathrm{cl} /$ actas/dnsc/documentService/ downloadWs?uuid=cd39e781-3561-4fbfalbe-a55f6d0c326f. 